\title{
ON NONSTATIONARY MOTION OF A FIXED MASS OF A GENERAL FLUID BOUNDED BY A FREE SURFACE
}

\author{
EWA ZADRZYŃSKA \\ Faculty of Mathematics and Information Sciences \\ Warsaw University of Technology \\ pl. Politechniki 1, 00-661 Warszawa, Poland \\ E-mail:emzad@impan.gov.pl \\ WOJCIECH M. ZAJĄCZKOWSKI* \\ Institute of Mathematics, Polish Academy of Sciences \\ Sniadeckich 8, P.O.Box 21, 00-956 Warszawa, Poland \\ E-mail:wz@impan.gov.pl
}

\begin{abstract}
In the paper the motion of a fixed mass of a viscous compressible heat conducting fluid is considered. Assuming that the initial data are sufficiently close to an equilibrium state and the external force, the heat sources and the heat flow through the boundary vanish, we prove the existence of a global in time solution which is close to the equilibrium state for any moment of time.
\end{abstract}

1. Introduction. In this paper we examine the global motion of a drop of a viscous compressible heat conducting fluid in the general case, i.e. without assuming any conditions on the form of the internal energy per unit mass $e=e(\rho, \theta)$. Here $\rho=\rho(x, t)$ and $\theta=\theta(x, t)$ (where $x \in \Omega_{t}, t \in(0, T), \Omega_{t} \subset \mathbb{R}^{3}$ is a bounded domain of a drop at time $t$ ) are the density and the temperature of the drop, respectively.

Next, let $v=v(x, t)$ denote the velocity of the fluid, $p=p(\rho, \theta)$ the pressure, $c_{v}=$ $c_{v}(\rho, \theta)$ the specific heat at constant volume, $\mu$ and $\nu$ the constant viscosity coefficients, $\varkappa$ the constant coefficient of the heat conductivity, $p_{0}$ the external (constant) pressure.

2000 Mathematics Subject Classification: 35Q35, 35R35, 76N10.

Key words and phrases: free boundary compressible viscous heat-conducting fluid, global existence.

Research supported by KBN grant No. 2P03A03816.

* Current address: Interdisciplinary Centre for Mathematical and Computational Modelling, Warsaw University, Pawińskiego 5a, 02-106 Warszawa, Poland.

The paper is in final form and no version of it will be published elsewhere. 
Then the motion of the drop is described by the following system of equations (see [1], [2]):

$$
\begin{array}{ll}
\rho\left[v_{t}+(v \cdot \nabla) v\right]-\operatorname{div} \mathbb{T}(v, p)=0 & \text { in } \tilde{\Omega}^{T}, \\
\rho_{t}+\operatorname{div}(\rho v)=0 & \text { in } \tilde{\Omega}^{T}, \\
\rho c_{v}\left(\theta_{t}+v \cdot \nabla \theta\right)-\varkappa \Delta \theta+\theta p_{\theta} \operatorname{div} v & \\
\quad-\frac{\mu}{2} \sum_{i, j=1}^{3}\left(v_{i x_{j}}+v_{j x_{i}}\right)^{2}-(\nu-\mu)(\operatorname{div} v)^{2}=0 & \text { in } \tilde{\Omega}^{T}, \\
\mathbb{T} \bar{n}=-p_{0} \bar{n} & \text { on } \tilde{S}^{T}, \\
v \cdot \bar{n}=-\frac{\varphi_{t}}{|\nabla \varphi|} & \text { on } \tilde{S}^{T}, \\
\frac{\partial \theta}{\partial n}=0 & \text { on } \tilde{S}^{T}, \\
\left.\rho\right|_{t=0}=\rho_{0},\left.\quad v\right|_{t=0}=v_{0},\left.\quad \theta\right|_{t=0}=\theta_{0} & \text { in } \Omega,
\end{array}
$$

where $\tilde{\Omega}^{T}=\bigcup_{t \in(0, T)} \Omega_{t} \times\{t\}, \tilde{S}^{T}=\bigcup_{t \in(0, T)} S_{t} \times\{t\}, S_{t}=\partial \Omega_{t} ; \varphi(x, t)=0$ describes $S_{t}$ (at least locally), $\bar{n}$ is the unit outward vector normal to the boundary, i.e. $\bar{n}=\frac{\nabla \varphi}{|\nabla \varphi|}$, $\Omega=\left.\Omega_{t}\right|_{t=0}=\Omega_{0}$. In (1.1) $\mathbb{T}=\mathbb{T}(v, p)=\left\{T_{i j}\right\}_{i, j=1,2,3}=\left\{2 \mu S_{i j}(v)+(\nu-\mu) \delta_{i j} \operatorname{div} v-\right.$ $\left.p \delta_{i j}\right\}_{i, j=1,2,3}$, where $S(v)=\left\{S_{i j}(v)\right\}_{i, j=1,2,3}=\left\{\frac{1}{2}\left(v_{i x_{j}}+v_{j x_{i}}\right)\right\}_{i, j=1,2,3}$.

Finally, we assume that $p_{\rho}>0, p_{\theta}>0$ for $\rho>0, \theta>0$.

Let us introduce the Lagrangian coordinates as the initial data to the Cauchy problem

$$
\frac{d x}{d t}=v(x, t),\left.\quad x\right|_{t=0}=\xi \in \Omega .
$$

Then, we obtain the following relation between the Eulerian $x$ and the Lagrangian $\xi$ coordinates of the same fluid particle:

$$
x=\xi+\int_{0}^{t} u\left(\xi, t^{\prime}\right) d t^{\prime} \equiv X_{u}(\xi, t),
$$

where $u(\xi, t)=v\left(X_{u}(\xi, t), t\right)$.

Let $\Omega$ be given. Then by $(1.1)_{5}, \Omega_{t}=\left\{x \in \mathbb{R}^{3}: x=X_{u}(\xi, t), \xi \in \Omega\right\}$ and $S_{t}=$ $\left\{x \in \mathbb{R}^{3}: x=X_{u}(\xi, t), \xi \in S=\partial \Omega\right\}$. By the continuity equation (1.1) 2 and the kinematic condition $(1.1)_{5}$ the total mass in conserved, i.e.

$$
\int_{\Omega_{t}} \rho(x, t) d x=\int_{\Omega} \rho_{0}(\xi) d \xi \equiv M
$$

where $M$ is a given constant.

The aim of this paper is to prove the existence of a global-in-time solution to problem (1.1). For this purpose we have to introduce an equilibrium state.

Definition 1.1. By an equilibrium state we mean a solution $\left(v, \rho, \theta, \Omega_{t}\right)$ of (1.1) such that $v=0, \theta=\theta_{e}, \rho=\rho_{e}, \Omega_{t}=\Omega_{e}$ for $t \geq 0$, where $\rho_{e}, \theta_{e}$ are positive constants satisfying the state equation

$$
p\left(\rho_{e}, \theta_{e}\right)=p_{0},
$$

and $\Omega_{e}$ is a domain of volume $\left|\Omega_{e}\right|=\frac{M}{\rho_{e}}$. 
To prove the global-in-time existence of solutions to problem (1.1) we have to restrict to looking for a solution which is close to the equilibrium state. Therefore we introduce

$$
p_{\sigma}=p-p_{0}, \quad \theta_{\sigma}=\theta-\theta_{e}, \quad \rho_{\sigma}=\rho-\rho_{e} .
$$

The paper consists of three sections. In Section 2 we present notation and auxiliary results, i.e. the local existence theorem for problem (1.1) proved in [11] and a differential inequality for the local solution proved in [13].

Section 3 contains a few auxiliary lemmas and the main result of the paper - Theorem 3.7 which yields the global existence of solutions of problem (1.1), close to the equilibrium state.

The global motion of a viscous compressible heat-conducting fluid bounded by a free surface in $\mathbb{R}^{3}$ has been also considered in papers [7]-[10].

In [10] we proved the existence of a global solution $(v, \theta, \rho)$ to problem (1.1) such that $\phi(t)+\int_{0}^{t} \Phi\left(t^{\prime}\right) d t^{\prime}<\infty$, where $t \in \mathbb{R}_{+}^{1}, \phi(t)=|v|_{3,0, \Omega_{t}}^{2}+\left|\theta_{\sigma}\right|_{3,0, \Omega_{t}}^{2}+\left|\rho_{\sigma}\right|_{3,0, \Omega_{t}}^{2}$, $\Phi(t)=|v|_{4,1, \Omega_{t}}^{2}+\left|\theta_{\sigma}\right|_{4,1, \Omega_{t}}^{2}+\left|\rho_{\sigma}\right|_{3,0, \Omega_{t}}^{2}$, the norms $|u|_{l, k, \Omega_{t}}$ for $u \in\left\{v, \theta_{\sigma}, \rho_{\sigma}\right\}$ are defined in Section 2. The global existence in [10] was proved under the assumption of a special form of internal energy $e(\rho, \theta)$ per unit mass.

Analogous result for equations describing the motion of a viscous compressible heatconducting capillary fluid but without assuming any conditions on the form of the internal energy $e$ has been proved in [7].

Paper [8] is also concerned with the free boundary problem (1.1) in the case when the shape of a free boundary is governed by a surface tension. It contains a proof of the global existence of solutions such that $\left(u, \vartheta_{\sigma}, \eta_{\sigma}\right) \in W_{2}^{2+\alpha, 1+\alpha / 2}\left(\Omega_{k T} \times(k T, t)\right) \times$ $W_{2}^{2+\alpha, 1+\alpha / 2}\left(\Omega_{k T} \times(k T, t)\right) \times C\left([k T, t] ; W_{2}^{1+\alpha}\left(\Omega_{k T}\right)\right) \cap W_{2}^{1+\alpha, 1 / 2+\alpha / 2}\left(\Omega_{k T} \times(k T, t)\right)$, where $u, \vartheta_{\sigma}, \eta_{\sigma}$ denote $v, \theta_{\sigma}, \rho_{\sigma}$ written in the Lagrangian coordinates $\xi \in \Omega_{k T} ; \alpha \in\left(\frac{3}{4}, 1\right), k T \leq$ $t \leq(k+1) T, k \in \mathbb{N} \cup\{0\} ; W_{2}^{2+\alpha, 1+\alpha / 2}\left(\Omega_{k T} \times(k T, t)\right)$ and $W_{2}^{1+\alpha, 1 / 2+\alpha / 2}\left(\Omega_{k T} \times(k T, t)\right)$ denote the anisotropic Sobolev-Slobodetskii spaces.

Papers [6], [12], [14], [15] are concerned with the global existence theorems for free boundary problems for equations of the motion of viscous compressible barotropic fluids.

Papers [3]-[5] are devoted to the global motion of a viscous incompressible fluids bounded by a free surface, both with the surface tension $([3],[4])$ and without it ([5]).

2. Notation and auxiliary results. First, we introduce some notation. Let

$$
\begin{aligned}
\|u\|_{k, \Omega} & =\|u\|_{H^{k}(\Omega)}, & & k \in \mathbb{N} ; \\
|u|_{p, \Omega} & =\|u\|_{L_{p}(\Omega)}, & & p \in[0, \infty] ; \\
|u(t)|_{k, l, \Omega} & =\sum_{i=0}^{k-l}\left\|\partial_{t}^{i} u(t)\right\|_{k-i, \Omega}, & & k, l \in \mathbb{N} .
\end{aligned}
$$

Let us introduce the spaces

$$
\begin{gathered}
\mathcal{A}_{T, \Omega_{i T}}=L_{2}\left(i T,(i+1) T ; H^{3}\left(\Omega_{i T}\right)\right) \cap \mathcal{B}_{T, \Omega_{i T}}, \\
\mathcal{B}_{T, \Omega_{i T}}=\bigcap_{j=0}^{2} C^{j}\left([i T,(i+1) T] ; H^{2-j}\left(\Omega_{i T}\right)\right) \cap \bigcap_{j=1}^{2} L_{2}^{j}\left(i T,(i+1) T ; H^{3-j}\left(\Omega_{i T}\right)\right),
\end{gathered}
$$


where $\Omega_{i T}$ is the considered domain at time $t=i T, i \in \mathbb{N} \cup\{0\}$ and

$$
\begin{aligned}
C^{j}\left(\left[T_{1}, T_{2}\right] ; X\right) & =\left\{u: \partial_{t}^{j} u \in C\left(\left[T_{1}, T_{2}\right] ; X\right)\right\}, \\
L_{2}^{j}\left(T_{1}, T_{2} ; X\right) & =\left\{u: \partial_{t}^{j} u \in L_{2}\left(T_{1}, T_{2} ; X\right)\right\} .
\end{aligned}
$$

We define

$$
\mathcal{A}_{T, \Omega_{0 T}} \equiv \mathcal{A}_{T, \Omega} \quad \text { and } \quad \mathcal{B}_{T, \Omega_{0 T}} \equiv \mathcal{B}_{T, \Omega} .
$$

In order to formulate the local existence theorem rewrite problem (1.1) in the Lagrangian coordinates as follows:

$$
\begin{array}{ll}
\eta u_{t}-\operatorname{div}_{u} \mathbb{T}_{u}(u, p)=0 & \text { in } \Omega^{T}, \\
\eta_{t}+\eta \operatorname{div}_{u} u=0 & \text { in } \Omega^{T}, \\
\eta c_{v} \vartheta_{t}+\vartheta p_{\vartheta} \operatorname{div}_{u} u-\varkappa \nabla_{u}^{2} \vartheta & \\
\quad-\frac{\mu}{2} \sum_{i, j=1}^{3}\left(\xi_{x_{i}} \cdot \nabla_{\xi} u_{j}+\xi_{x_{j}} \cdot \nabla_{\xi} u_{i}\right)^{2}-(\nu-\mu)\left(\operatorname{div}_{u} u\right)^{2}=0 & \text { in } \Omega^{T}, \\
\mathbb{T}_{u}(u, p) \bar{n}_{u}=-p_{0} \bar{n}_{u} & \text { on } S^{T}, \\
\bar{n}_{u} \cdot \nabla_{u} \vartheta=0 & \text { on } S^{T}, \\
\left.u\right|_{t=0}=v_{0},\left.\vartheta\right|_{t=0}=\theta_{0},\left.\quad \eta\right|_{t=0}=\rho_{0} & \text { in } \Omega,
\end{array}
$$

where $\Omega^{T} \equiv \Omega \times(0, T), S^{T} \equiv S \times(0, T), u(\xi, t)=v\left(X_{u}(\xi, t), t\right) ; \vartheta(\xi, t)=\theta\left(X_{u}(\xi, t), t\right)$; $\eta(\xi, t)=\rho\left(X_{u}(\xi, t), t\right) ; \bar{n}_{u}(\xi, t)=\bar{n}\left(X_{u}(\xi, t), t\right) ; X_{u}$ is given by $(1.2) ; \nabla_{u}=\xi_{i x} \partial_{\xi_{i}}=$ $\left\{\xi_{i x_{j}} \partial_{\xi_{i}}\right\}_{j=1,2,3}, \mathbb{T}_{u}(u, p)=\left\{\mu\left(\partial_{x_{i}} \xi_{k} \partial_{\xi_{k}} u_{j}+\partial_{x_{j}} \xi_{k} \partial_{\xi_{k}} u_{i}\right)+(\nu-\mu) \delta_{i j} \operatorname{div}_{u} u-p \delta_{i j}\right\}_{i, j=1,2,3}$, $\operatorname{div}_{u} u=\nabla_{u} \cdot u=\partial_{x_{i}} \xi_{k} \partial_{\xi_{k}} u_{i}, \operatorname{div}_{u} \mathbb{T}_{u}(u, p)=\left\{\partial_{x_{j}} \xi_{k} \partial_{\xi_{k}} T_{u i j}(u, p)\right\}_{i, j=1,2,3}$ and $\partial_{x_{i}} \xi_{k}$ are elements of matrix $\xi_{x}$ which is inverse to the matrix $x_{\xi}=I+\int_{0}^{t} u_{\xi}\left(\xi, t^{\prime}\right) d t^{\prime}$.

To prove the local existence of solutions we apply the method of successive approximations taking as zero step functions $u_{0} \in W_{2}^{2+\alpha, 1+\alpha / 2}\left(\Omega^{T}\right)$ and $\vartheta_{0} \in W_{2}^{2+\alpha, 1+\alpha / 2}\left(\Omega^{T}\right)$ which are solutions of the following parabolic problems:

$$
\begin{array}{ll}
u_{0 t}-\operatorname{div} \mathbb{D}\left(u_{0}\right)=0 & \text { in } \Omega^{T}, \\
\mathbb{D}\left(u_{0}\right) \bar{n}_{0}=\left(p\left(\rho_{0}, \theta_{0}\right)-p_{0}\right) \bar{n}_{0} & \text { on } S^{T}, \\
\left.u_{0}\right|_{t=0}=v_{0} & \text { in } \Omega,
\end{array}
$$

(where $\left.\mathbb{D}\left(u_{0}\right)=\left\{2 \mu S_{i j}\left(u_{0}\right)+(\nu-\mu) \delta_{i j} \operatorname{div} u_{0}\right\}_{i, j=1,2,3}\right)$ and

$$
\begin{array}{ll}
\vartheta_{0 t}-\varkappa \Delta \vartheta_{0}=0 & \text { in } \Omega^{T}, \\
\bar{n}_{0} \cdot \nabla_{\xi} \vartheta_{0}=0 & \text { on } S^{T}, \\
\left.\vartheta_{0}\right|_{t=0}=\theta_{0} & \text { in } \Omega,
\end{array}
$$

where $\bar{n}_{0}$ is the unit outward vector normal to $S$.

Functions $u_{0}$ and $\vartheta_{0}$ satisfy the estimates (see [11], estimates (4.3) and (4.5)):

$$
\begin{aligned}
\left\|u_{0}\right\|_{\mathcal{A}_{T, \Omega}}^{2} \leq C_{1}(T)\left[\| p\left(\rho_{0}, \theta_{0}\right)-p_{0}\right) & \bar{n}_{0}\left\|_{3 / 2, S}^{2}+\right\| v_{0} \|_{2, \Omega}^{2} \\
& \left.+\left\|u_{0 t}(0)\right\|_{1, \Omega}^{2}+\left\|u_{0 t t}(0)\right\|_{0, \Omega}^{2}\right] \equiv F_{1}(t)
\end{aligned}
$$

and

$$
\left\|\vartheta_{0}\right\|_{\mathcal{A}_{T, \Omega}}^{2} \leq c_{2}(T)\left(\left\|\theta_{0}\right\|_{2, \Omega}^{2}+\left\|\vartheta_{0 t}(0)\right\|_{1, \Omega}^{2}+\left\|\vartheta_{0 t t}(0)\right\|_{0, \Omega}^{2}\right) \equiv F_{2}(T)
$$


where $C_{1}$ and $C_{2}$ are positive continuous increasing functions of $T ; u_{0 t}(0), u_{0 t t}(0), \theta_{0 t}(0)$, $\vartheta_{0 t t}(0)$ are calculated from $(2.2)$ and $(2.3)$.

Finally, $\eta_{0}$ is a solution of the problem

$$
\begin{array}{ll}
\eta_{0 t}+\eta_{0} \nabla_{u_{0}} \cdot u_{0}=0 & \text { in } \Omega^{T}, \\
\left.\eta_{0}\right|_{t=0}=\rho_{0} & \text { in } \Omega .
\end{array}
$$

Next, assume that

$$
\begin{gathered}
\rho_{1}<\rho_{0}<\rho_{2}, \quad \theta_{1}<\theta_{0}<\theta_{2} \quad \text { for all } \xi \in \Omega \\
\sigma_{1}<\frac{1}{c_{v}(\rho, \theta)}<\sigma_{2} \quad \text { for } \rho \in\left(\rho_{1}, \rho_{2}\right), \theta \in\left(\theta_{1}, \theta_{2}\right)
\end{gathered}
$$

and define

$$
\begin{aligned}
H_{0}=\sigma_{2}+ & \frac{1}{\rho_{1}}+\left\|\rho_{0}\right\|_{2, \Omega}^{2}+\left\|v_{0}\right\|_{2, \Omega}^{2}+\left\|\theta_{0}\right\|_{2, \Omega}^{2} \\
& +\left\|u_{t}(0)\right\|_{1, \Omega}^{2}+\left\|\vartheta_{t}(0)\right\|_{1, \Omega}^{2}+\left\|u_{t t}(0)\right\|_{0, \Omega}^{2}+\left\|\vartheta_{t t}(0)\right\|_{0, \Omega}^{2}<\tilde{H}_{0}
\end{aligned}
$$

where $u_{t}(0), u_{t t}(0), \vartheta_{t}(0), \vartheta_{t t}(0)$ are calculated from $(1.1)_{1}$ and $(1.1)_{2}$, respectively; $\tilde{H}_{0}>0$ is a constant. Then the following theorem holds.

Theorem 2.1 (see [11], Theorem 4.2). Assume that $v_{0}, \rho_{0}, \theta_{0} \in H^{2}(\Omega) ; \rho_{0}, \theta_{0}>0$; $u_{t}(0), \vartheta_{t}(0), u_{0 t}(0), \vartheta_{0 t}(0) \in H^{1}(\Omega) ; u_{t t}(0), \vartheta_{t t}(0), u_{0 t t}(0), \vartheta_{0 t t}(0) \in L^{2}(\Omega) ; S \in H^{5 / 2}$, $p \in C^{3}\left(\mathbb{R}^{2}\right), c_{v} \in C^{2}\left(\mathbb{R}^{2}\right), c_{v}>0$. Let assumptions (2.6), (2.7) and the following compatibility conditions be satisfied:

$$
\begin{gathered}
\mathbb{D}\left(v_{0}\right) \bar{n}_{0}=\left(p\left(\rho_{0}, \theta_{0}\right)-p_{0}\right) \bar{n}_{0} \quad \text { on } S, \\
\bar{n}_{0} \cdot \nabla_{\xi} \theta_{0}=0 \quad \text { on } S .
\end{gathered}
$$

Assume that $F_{1}(t)+F_{2}(t)<A$ for $t \leq T$, where $A>0$ is a constant depending also on $\tilde{H}_{0}$ (i.e. there exists a positive continuous increasing function $F=F\left(\tilde{H}_{0}\right)$ satisfying $F\left(\tilde{H}_{0}\right)<A$ ). Then there exists $T>0$ (depending on $A$ ) such that there exists a unique solution $u, \vartheta \in \mathcal{A}_{T, \Omega}, \eta \in \mathcal{B}_{T, \Omega}$ of (2.1) and

$$
\begin{gathered}
\|u\|_{\mathcal{A}_{T, \Omega}}^{2}+\|\vartheta\|_{\mathcal{A}_{T, \Omega}}^{2} \leq A, \\
\|\eta\|_{\mathcal{B}_{T, \Omega}}^{2} \leq \psi_{1}(A),
\end{gathered}
$$

where $\psi_{1}$ is a positive continuous increasing function of $A$.

Now, in view of Lemmas 3.5, 3.6, 2.3 of [11] and Theorem 2.1 we obtain

Lemma 2.2. Let the assumptions of Theorem 2.1 be satisfied. Then for a sufficiently small time $T$ of the local existence, the local solution of problem (2.1) satisfies the estimate

$$
\begin{aligned}
\|u\|_{\mathcal{A}_{T, \Omega}}^{2} & +\left\|\vartheta_{\sigma}\right\|_{\mathcal{A}_{T, \Omega}}^{2}+\left\|\eta_{\sigma}\right\|_{\mathcal{B}_{T, \Omega}}^{2} \\
\leq & \psi_{2}(A, T)\left(\left\|v_{0}\right\|_{2, \Omega}^{2}+\left\|\theta_{\sigma 0}\right\|_{2, \Omega}^{2}+\left\|\rho_{\sigma 0}\right\|_{2, \Omega}^{2}+\left\|u_{t}(0)\right\|_{1, \Omega}^{2}\right. \\
& \left.\quad+\left\|u_{t t}(0)\right\|_{0, \Omega}^{2}+\left\|\vartheta_{t}(0)\right\|_{1, \Omega}^{2}+\left\|\vartheta_{t t}(0)\right\|_{0, \Omega}^{2}\right),
\end{aligned}
$$

where $\vartheta_{\sigma}=\vartheta-\theta_{e}, \eta_{\sigma}=\eta-\rho_{e}, \theta_{\sigma 0}=\theta_{0}-\theta_{e}, \rho_{\sigma 0}=\rho_{0}-\rho_{e} ; \theta_{e}$ and $\rho_{e}$ are given by Definition 1.1 . 
Now, we recall the differential inequality proved in [13]. To derive this inequality we use a partition of unity $\left(\left\{\tilde{\Omega}_{i}\right\},\left\{\zeta_{i}\right\}\right), \Omega \subset \bigcup_{i \in \mathcal{M} \cup \mathcal{N}} \tilde{\Omega}_{i}, \sum_{i \in \mathcal{M} \cup \mathcal{N}} \zeta_{i}(x)=1$ for $x \in \Omega$, where $\tilde{\Omega}_{i}, i \in \mathcal{M}$, are interior subdomains and $\tilde{\Omega}_{i}, i \in \mathcal{N}$, are boundary subdomains, i.e. $\overline{\tilde{\Omega}}_{i} \subset \Omega$ for $i \in \mathcal{M}$ and $\tilde{\Omega}_{i} \cap S \neq \varnothing$ for $i \in \mathcal{N}$. We can assume that $\zeta_{i}(\xi)=1$ for $\xi \in \tilde{\omega}_{i}$, where $\tilde{\omega}_{i}$ is such that $\overline{\tilde{\omega}}_{i} \subset \tilde{\Omega}_{i}$.

Consider now a boundary subdomain $\tilde{\Omega}_{i}$ (which we denote for simplicity by $\tilde{\Omega}$ ) and let $\beta \in \overline{\tilde{\omega}}_{i} \cap S \subset \tilde{\Omega} \cap S, \tilde{S}=S \cap \tilde{\Omega}$. Introduce local coordinates connected with $\{\xi\}$ by

$$
y_{k}=\alpha_{k l}\left(\xi_{l}-\beta_{l}\right), \quad \alpha_{3 k}=n_{k}(\beta), \quad k=1,2,3,
$$

where $\left\{\alpha_{k l}\right\}$ is a constant orthogonal matrix such that $\tilde{S}$ is determined by

$$
y_{3}=F\left(y_{1}, y_{2}\right), \quad F \in H^{5 / 2}
$$

and

$$
\tilde{\Omega} \cap \Omega=\left\{y:\left|y_{i}\right|<d, i=1,2, F\left(y^{\prime}\right)<y_{3}<F\left(y^{\prime}\right)+d, y^{\prime}=\left(y_{1}, y_{2}\right)\right\} .
$$

Next, we introduce functions $u^{\prime}, \vartheta^{\prime}$ and $\eta^{\prime}$ by

$$
u_{i}^{\prime}(y)=\left.\alpha_{i j} u_{j}(\xi)\right|_{\xi=\xi(y)}, \quad \vartheta^{\prime}(y)=\left.\vartheta(\xi)\right|_{\xi=\xi(y)}, \quad \eta^{\prime}(y)=\left.\eta(\xi)\right|_{\xi=\xi(y)},
$$

where $\xi=\xi(y)$ is the inverse transformation to (2.12).

Further we introduce the transformation of variables, $z=\Psi(y)$, by

$$
z_{i}=y_{i}, \quad i=1,2, \quad z_{3}=y_{3}-\tilde{F}(y), \quad y \in \tilde{\Omega},
$$

where $\tilde{F}$ is an extension of $F$ to $\tilde{\Omega}$.

Let

$$
\hat{\Omega}=\Psi(\tilde{\Omega} \cap \Omega)=\left\{z:\left|z_{i}\right|<d, i=1,2,0<z_{3}<d\right\}
$$

and $\hat{S}=\Psi(\tilde{S})$.

Define

$$
\hat{u}(z)=\left.u^{\prime}(y)\right|_{y=\Psi^{-1}(z)}, \quad \hat{\vartheta}(z)=\left.\vartheta^{\prime}(y)\right|_{y=\Psi^{-1}(z)}, \quad \hat{\eta}(z)=\left.\eta^{\prime}(y)\right|_{y=\Psi^{-1}(z)} .
$$

Let $\hat{\nabla}_{k}=\left.\xi_{l x_{k}} z_{i \xi_{l}} \nabla_{z_{i}}\right|_{\xi=\chi^{-1}(z)}$, where $\chi(\xi)=\Psi(\psi(\xi))$ and $y=\psi(\xi)$ is defined by $(2.12)$. Introduce also the notation

$$
\begin{aligned}
& \tilde{u}(\xi)=u(\xi) \zeta(\xi), \tilde{\vartheta}_{\sigma}(\xi)=\vartheta_{\sigma}(\xi) \zeta(\xi), \tilde{\eta}_{\sigma}(\xi)=\eta_{\sigma}(\xi) \zeta(\xi), \quad \xi \in \tilde{\Omega}, \tilde{\Omega} \cap S=\varnothing, \\
& \tilde{u}(z)=\hat{u}(z) \hat{\zeta}(z), \tilde{\vartheta}_{\sigma}(z)=\hat{\vartheta}_{\sigma}(z) \hat{\zeta}(z), \tilde{\eta}_{\sigma}(z)=\hat{\eta}_{\sigma}(z) \hat{\zeta}(z), \quad z \in \hat{\Omega}, \quad \tilde{\Omega} \cap S \neq \varnothing,
\end{aligned}
$$

where $\hat{\zeta}(z)=\left.\zeta(\xi)\right|_{\xi=\chi^{-1}(z)}$.

Next, assume that

$$
\rho_{1}<\rho(x, t)<\rho_{2}, \quad \theta_{1}<\theta(x, t)<\theta_{2}
$$

for $x \in \bar{\Omega}_{t}, t \in[0, T]$ (where $T$ is the time of the local existence) and introduce the functions:

$$
\begin{gathered}
\phi(t)=|v|_{2,0, \Omega_{t}}^{2}+\left|\theta_{\sigma}\right|_{2,0, \Omega_{t}}^{2}+\left|\rho_{\sigma}\right|_{2,0, \Omega_{t}}^{2} \\
\Phi(t)=|v|_{3,1, \Omega_{t}}^{2}+\left|\theta_{\sigma}\right|_{3,1, \Omega_{t}}^{2}+\left\|\rho_{\sigma}\right\|_{2, \Omega_{t}}^{2}+\left\|\rho_{\sigma t}\right\|_{2, \Omega_{t}}^{2}+\left\|\rho_{\sigma t t}\right\|_{1, \Omega_{t}}^{2},
\end{gathered}
$$




$$
\begin{aligned}
\bar{\phi}(t)= & \sum_{j=0}^{1} \int_{\Omega_{t}} \rho\left[\bar{a}_{j}\left(\partial_{t}^{j} v\right)^{2}+\bar{b}_{j}\left(\partial_{t}^{j} \rho_{\sigma}\right)^{2}+\bar{c}_{j}\left(\partial_{t}^{j} \theta_{\sigma}\right)^{2}\right] d x \\
+ & \int_{\Omega_{t}} \bar{d}\left(\rho v_{t t}^{2}+\frac{p_{\sigma \rho}}{\rho} \rho_{\sigma t t}^{2}+\frac{\rho c_{v}}{\theta} \theta_{\sigma t t}^{2}\right) d x \\
+ & \sum_{i \in \mathcal{M}}\left\{\int_{\tilde{\Omega}_{i}} \sum_{1 \leq|\alpha| \leq 2}\left[\eta\left(D_{\xi}^{\alpha} \tilde{u}\right)^{2}+\frac{p_{\sigma \eta}}{\eta}\left(D_{\xi}^{\alpha} \tilde{\eta}_{\sigma}\right)^{2}+\frac{\eta c_{v}}{\vartheta}\left(D_{\xi}^{\alpha} \tilde{\vartheta}_{\sigma}\right)^{2}\right] A d \xi\right. \\
& \left.+\int_{\tilde{\Omega}_{i}}\left(\eta \tilde{u}_{t \xi}^{2}+\frac{p_{\sigma \eta}}{\eta} \tilde{\eta}_{\sigma t \xi}^{2}+\frac{\eta c_{v}}{\vartheta} \tilde{\vartheta}_{\sigma t \xi}^{2}\right) A d \xi\right\} \\
+ & \sum_{i \in \mathcal{N}}\left\{\int_{\hat{\Omega}_{i}} \sum_{1 \leq|\alpha| \leq 2} \bar{e}_{\alpha}\left[\hat{\eta}\left(D_{\tau}^{\alpha} \tilde{u}\right)^{2}+\frac{p_{\sigma \hat{\eta}}}{\hat{\eta}}\left(D_{\tau}^{\alpha} \tilde{\eta}_{\sigma}\right)^{2}+\frac{\hat{\eta} c_{v}}{\hat{\vartheta}}\left(D_{\tau}^{\alpha} \tilde{\vartheta}_{\sigma}\right)^{2}\right] J d z\right. \\
& +\int_{\hat{\Omega}_{i}} \bar{e}\left(\hat{\eta} \tilde{u}_{t \tau}^{2}+\frac{p_{\sigma \hat{\eta}}}{\hat{\eta}} \tilde{\eta}_{\sigma t \tau}^{2}+\frac{\hat{\eta} c_{v}}{\hat{\vartheta}} \tilde{\vartheta}_{\sigma t \tau}^{2}\right) J d z \\
& +\int_{\hat{\Omega}_{i}} \sum_{0 \leq|\alpha| \leq 1}\left[\bar{f}_{\alpha} \frac{p_{\sigma \hat{\eta}}}{\hat{\eta}}\left(D_{\tau, t}^{\alpha} \tilde{\eta}_{\sigma n}\right)^{2}+\bar{g}_{\alpha} \hat{\eta}\left(D_{\tau, t}^{\alpha} \tilde{u}_{3 n}\right)^{2}\right. \\
& \left.\left.+\bar{h}_{\alpha} \frac{\hat{\eta} c_{v}}{\hat{\vartheta}}\left(D_{\tau, t}^{\alpha} \tilde{\vartheta}_{\sigma n}\right)^{2}\right] J d z+\int_{\hat{\Omega}_{i}}\left(\frac{p_{\sigma \hat{\eta}}}{\hat{\eta}} \tilde{\eta}_{\sigma n n}^{2}+\frac{\hat{\eta} c_{v}}{\hat{\vartheta}} \tilde{\vartheta}_{\sigma n n}^{2}\right) J d z\right\} \\
+ & \int_{\Omega} \eta\left(u_{\xi}^{2}+u_{t \xi}^{2}+u_{\xi \xi}^{2}\right) A d \xi,
\end{aligned}
$$

where $\bar{a}_{j}, \bar{b}_{j}, \bar{c}_{j}(j=0,1), \bar{d}, \bar{e}_{\alpha}(\alpha: 1 \leq|\alpha| \leq 2), \bar{e}, \bar{f}_{\alpha}, \bar{g}_{\alpha}, \bar{h}_{\alpha}(\alpha: 0 \leq|\alpha| \leq 1)$ are positive constants depending on $\rho_{1}, \rho_{2}, \theta_{1}, \theta_{2}, \mu, \nu, \varkappa, c_{v}, p,\|S\|_{5 / 2}, T, \int_{0}^{T}\|v\|_{3, \Omega_{t}}^{2} d t$ and the constants from the imbedding theorems and the Korn inequalities (which depend on $\left.\Omega_{t}, t \leq T\right) ; A$ and $J$ are the Jacobians of transformations $x=x(\xi)$ and $x=x(z)$, respectively.

By $\tau$ we denoted in $(2.16) z_{1}, z_{2}$, i.e. $\tau=\left(z_{1}, z_{2}\right)$ and by $n$ we denoted $z_{3}$. Moreover, $\alpha$ is a multiindex and

$$
\begin{array}{ll}
D_{\xi}^{\alpha} f=\frac{\partial^{|\alpha|} f}{\partial \xi_{1}^{\alpha_{1}} \partial \xi_{2}^{\alpha_{2}} \partial \xi_{3}^{\alpha_{3}}}, & D_{\tau}^{\alpha} f=\frac{\partial^{|\alpha|} f}{\partial z_{1}^{\alpha_{1}} \partial z_{2}^{\alpha_{2}}} \\
D_{t, \tau}^{\alpha} f=\frac{\partial^{|\alpha|} f}{\partial z_{1}^{\alpha_{1}} \partial z_{2}^{\alpha_{2}} \partial t^{\alpha_{3}}}, & |\alpha|=\sum_{i} \alpha_{i}, \quad f \in\left\{\tilde{u}, \tilde{\eta}_{\sigma}, \tilde{\vartheta}_{\sigma}\right\} .
\end{array}
$$

The following lemma holds.

Lemma 2.3 (see [13], Theorem 2). Let $\nu>\frac{1}{3} \mu>0, c_{v}>0, \varkappa>0, c_{v} \in C^{2}\left(\mathbb{R}^{2}\right)$, $p \in C^{2}\left(\mathbb{R}^{2}\right), p_{\rho}>0, p_{\theta}>0$ for $\rho, \theta>0$ and let assumption (2.13) be satisfied. Moreover, assume that

$$
\int_{\Omega} \rho_{0} v_{0} \cdot(a+b \times \xi) d \xi=0
$$

where $a$ and $b$ are arbitrary constant vectors.

Then for the local solution $(v, \theta, \rho)$ of problem (1.1) such that $u, \vartheta_{\sigma} \in \mathcal{A}_{T, \Omega}$ and 
$\eta_{\sigma} \in \mathcal{B}_{T, \Omega}$, the following differential inequality is satisfied

$$
\frac{d \bar{\phi}}{d t}+c_{0} \Phi \leq c_{1}\left[\phi\left(1+\phi^{2}\right)+\int_{0}^{t}\|v\|_{3, \Omega_{t^{\prime}}}^{2} d t^{\prime}\right] \Phi \quad \text { for } t \leq T
$$

where $T$ is the time of the local existence, $c_{0}$ and $c_{1}$ are positive constants depending on the same quantities as constants $\bar{a}_{j}, \bar{b}_{j}, \bar{c}_{j}(j=0,1), \bar{d}, \bar{e}_{\alpha}(1 \leq|\alpha| \leq 2), \bar{e}, \bar{f}_{\alpha}, \bar{g}_{\alpha}, \bar{h}_{\alpha}$ $(0 \leq|\alpha| \leq 1)$ from formula $(2.16)$.

3. Global existence. Let us introduce the spaces

$$
\begin{gathered}
\mathcal{N}(t)=\left\{\left(v, \theta_{\sigma}, \rho_{\sigma}\right): \phi(t)<\infty\right\}, \\
\mathcal{M}(t)=\left\{\left(v, \theta_{\sigma}, \rho_{\sigma}\right): \phi(t)+\int_{0}^{t} \Phi\left(t^{\prime}\right) d t^{\prime}<\infty\right\},
\end{gathered}
$$

where $\phi$ and $\Phi$ are given by (2.14) and (2.15), respectively.

From the definition of $\bar{\phi}$ (see $(2.16)$ ) it follows that

$$
c_{2} \phi(t) \leq \bar{\phi}(t) \leq c_{3} \phi(t) \text { for } t \leq T,
$$

where $c_{2}, c_{3}>0$ are constants depending on the same quantities as constants $c_{0}$ and $c_{1}$ from inequality (2.17).

In view of (3.1), estimate (2.11) from Lemma 2.2 yields

$$
\phi(t)+\int_{0}^{t} \Phi\left(t^{\prime}\right) d t^{\prime} \leq c_{4} \bar{\phi}(0)
$$

where $c_{4}>0$ is a constant depending on the same quantities as $c_{2}$ and $c_{3}$ and on $A$.

Hence we obtain the lemma.

Lemma 3.1. Let $\left(v, \theta_{\sigma}, \rho_{\sigma}\right) \in \mathcal{N}(0), S \in H^{5 / 2}, u_{0 t}, \vartheta_{0 t} \in H^{1}(\Omega), u_{0 t t}, \vartheta_{0 t t} \in L_{2}(\Omega)$ $\left(u_{0}, \vartheta_{0}\right.$ are solutions of problems $(2.2)$ and $(2.3)$, respectively $), c_{v} \in C^{2}\left(\mathbb{R}^{2}\right), c_{v}>0$, $p \in C^{3}\left(\mathbb{R}^{2}\right)$. Let assumptions (2.13), (2.7) and compatibility conditions (2.9), (2.10) be satisfied. Moreover, assume

$$
\phi(0) \leq \varepsilon
$$

Then the local solution $(v, \theta, \rho)$ of problem (1.1) is such that $\left(v, \theta_{\sigma}, \rho_{\sigma}\right) \in \mathcal{M}(t)$ for $t \leq T$ (where $T>0$ is the time of the local existence) and the following estimate holds

$$
\phi(t)+\int_{0}^{t} \Phi\left(t^{\prime}\right) d t^{\prime} \leq \frac{c_{4} c_{3}}{c_{2}} \varepsilon \quad \text { for } t \leq T .
$$

Another consequence of Lemma 2.2 is the remark.

REMARK 3.2. Estimate (2.11) and assumption (3.2) yield

$$
\begin{aligned}
& \left|\int_{0}^{t} u\left(\xi, t^{\prime}\right) d t^{\prime}\right| \leq c_{5} T^{1 / 2}\left(\int_{0}^{T}\|u\|_{2, \Omega}^{2} d t^{\prime}\right)^{1 / 2} \\
& \quad \leq c_{5} T^{1 / 2}\left[\psi_{2}(A, T) \phi(0)\right]^{1 / 2} \leq \frac{c_{5} c_{3}}{c_{2}} T^{1 / 2} \psi_{2}^{1 / 2}(A, T) \varepsilon^{1 / 2} \equiv c_{6} T^{1 / 2} \varepsilon^{1 / 2}
\end{aligned}
$$

where $\psi_{3}$ is a positive continuous function, $c_{5}>0$ is a constant from the imbedding theorem depending on $\Omega$. 
Hence, relation (1.2) implies that both the shape and the volume of $\Omega_{t}$ do not change much for $t \leq T$ and the constants $c_{i}(i=0, \ldots, 6)$ can be chosen independently of time for $t \leq T$.

Now, we prove

LEMMA 3.3. Let the assumptions of Lemmas 2.3 and 3.1 be satisfied. Then there exists a constant $\mu_{0}>0$ (depending on the same quantities as $c_{0}, \ldots, c_{6}$ ) such that

$$
\bar{\phi}(t) \leq \bar{\phi}(0) e^{-\mu_{0} t} \quad \text { for } t \leq T
$$

where $T>0$ is the time of the local existence.

Proof. For $\varepsilon$ sufficiently small inequalities (2.17) and (3.3) yield

$$
\frac{d \bar{\phi}}{d t}+c_{7} \Phi \leq 0
$$

Since $\bar{\phi} \leq c_{3} \Phi$ (where $c_{3}>0$ is the constant from (3.1)), we get (3.4) with $\mu_{0}=c_{7} / c_{3}$.

This concludes the proof.

Corollary 3.4. By estimate (3.1) inequality (3.4) yields

$$
\phi(t) \leq \frac{c_{3}}{c_{2}} \phi(0) e^{-\mu_{0} t} \quad \text { for } t \leq T .
$$

Lemma 3.3 suggests that the solution can be continued to the interval $[T, 2 T]$. However, to do this we must have the sum of the right-hand sides of (2.4) and (2.5) with initial conditions at $T$ estimated by $A$.

Let

$$
\begin{array}{ll}
g_{1}(t)=\left|u_{0}(t)\right|_{2,0, \Omega}^{2}, & G_{1}(t)=\left|u_{0}(t)\right|_{3,1, \Omega}^{2}-\left\|u_{0}(t)\right\|_{3, \Omega}^{2}, \\
g_{2}(t)=\left|\vartheta_{0}(t)\right|_{2,0, \Omega}^{2}-\left|\vartheta_{0}(t)\right|_{0, \Omega}^{2}, & G_{2}(t)=\left|\vartheta_{0}(t)\right|_{3,1, \Omega}^{2},
\end{array}
$$

where $u_{0}$ and $\vartheta_{0}$ are solutions of (2.2) and (2.3), respectively.

Lemma 3.5. Let assumption (3.2) be satisfied. Moreover, let

$$
g_{1}(0) \leq \alpha_{1},
$$

where $\alpha_{1}>0$ is a constant (not necessarily small). Then for $\varepsilon$ sufficiently small we have

$$
g_{1}(t) \leq \alpha_{1} \quad \text { for } t \leq T
$$

PROOF. Repeating the argument from [12] (see Lemma 3.8) we obtain the inequality

$$
\frac{1}{2} \frac{d}{d t} g_{1}(t)+c_{8} G_{1}(t) \leq c_{9}\left\|\rho_{\sigma 0}\right\|_{0, \Omega}^{2} G_{1}(t)+c_{10}\left(\left\|\rho_{\sigma 0}\right\|_{1, \Omega}^{2}+\left\|\theta_{\sigma 0}\right\|_{1, \Omega}^{2}\right)
$$

By assumption (3.2), estimate (3.5) yields

$$
\frac{1}{2} \frac{d}{d t} g_{1}(t)+\frac{c_{8}}{2} G_{1}(t) \leq c_{10} \varepsilon
$$

for $\varepsilon$ so small that $c_{9} \varepsilon \leq c_{8} / 2$.

Let $t_{*}=\inf \left\{t \in[0, T]: g_{1}(t)>\alpha_{1}\right\}$. Then $g_{1}(t)>\alpha_{1}$ in an interval $\left(t_{*}, t\right)$, where $t \leq T$. Integrating (3.6) over $\left(t_{*}, \tau\right)(\tau \leq t)$ and using the estimate $G_{1} \geq g_{1}$ we get

$$
g_{1}(t)+c_{8} \int_{t_{*}}^{\tau} g_{1}\left(t^{\prime}\right) d t^{\prime} \leq g_{1}\left(t_{*}\right)+2 c_{10} \varepsilon\left(\tau-t_{*}\right) .
$$


Hence

$$
g_{1}(t)+c_{8} \alpha_{1}\left(\tau-t_{*}\right) \leq \alpha_{1}+2 c_{10} \varepsilon\left(\tau-t_{*}\right) .
$$

Assuming that $\varepsilon$ is so small that $2 c_{10} \varepsilon \leq c_{8} \alpha_{1}$ we obtain

$$
g_{1}(\tau) \leq \alpha_{1} \quad \text { for } t_{*}<\tau \leq t
$$

a contradiction.

This completes the proof.

LEMMA 3.6. Let

$$
g_{2}(0) \leq \alpha_{2}
$$

where $\alpha_{2}>0$ is a constant. Then

$$
g_{2}(t) \leq \alpha_{2} \quad \text { for } t \leq T
$$

The proof of Lemma 3.6 is analogous to that of Lemma 3.5.

Now, we prove the main result of the paper.

Theorem 3.7. Let $\nu>\frac{1}{3} \mu>0, \varkappa>0, c_{v}>0, c_{v} \in C^{2}\left(\mathbb{R}^{2}\right), p \in C^{3}\left(\mathbb{R}^{2}\right) ; p_{\rho}>0$, $p_{\theta}>0$ for $\rho, \theta>0$ and assume that there exist $\rho_{e}>0$ and $\theta_{e}>0$ satisfying equation (1.3). Let $\left(v, \theta_{\sigma} \rho_{\sigma}\right) \in \mathcal{N}(0), S \in H^{5 / 2} ; u_{0 t}(0), \vartheta_{0 t}(0) \in H^{1}(\Omega) ; u_{0 t t}(0) \vartheta_{0 t t}(0) \in L_{2}(\Omega)\left(u_{0}, \vartheta_{0}\right.$ are solutions of (2.2) and (2.3), respectively) and let the following compatibility conditions be satisfied:

$$
\begin{gathered}
{\left[\mathbb{D}\left(v_{0}\right)-\left(p\left(\rho_{0}, \theta_{0}\right)-p_{0}\right)\right] \bar{n}_{0}=0 \quad \text { on } S,} \\
\bar{n}_{0} \cdot \nabla_{\xi} \theta_{0}=0 \quad \text { on } S,
\end{gathered}
$$

where $\bar{n}_{0}$ is the unit outward vector normal to $S$.

Moreover, assume that

$$
\phi(0) \leq \varepsilon
$$

$l>0$ is a constant such that

$$
\rho_{e}-l>0, \theta_{e}-l>0 \text { and } \rho_{1}<\rho_{0}<\rho_{2}, \quad \theta_{1}<\theta_{0}<\theta_{2},
$$

where $\rho_{1}=\rho_{e}-l, \rho_{2}=\rho_{e}+l, \theta_{1}=\theta_{e}-l, \theta_{2}=\theta_{e}-l\left(\rho_{e}, \theta_{e}\right.$ are introduced by Definition 1.1);

$$
\int_{\Omega} \rho_{0} v_{0} \cdot(a+b \times \xi) d \xi=0
$$

where $a$ and $b$ are arbitrary constant vectors;

$$
\int_{\Omega} \rho_{0} d \xi=M
$$

Then for sufficiently small $\varepsilon$ there exists a global solution of $(1.1)$ such that $\left(v, \theta_{\sigma}, \rho_{\sigma}\right) \in$ $\mathcal{M}(t)$ for $t \in \mathbb{R}_{+}, S_{t} \in H^{5 / 2}$ for $t \in \mathbb{R}_{+}$and

$$
\phi(t) \leq \frac{c_{3}}{c_{2}} \varepsilon \quad \text { for } t \in \mathbb{R}_{+},
$$

where $c_{2}$ and $c_{3}$ are constants from (3.10). 
ProOF. We prove the theorem as in the barotropic case (see [12], Theorem 3.9), step by step using the local existence in a fixed interval. First, notice that by (2.14) and (2.16),

$$
c_{2} \phi(0) \leq \bar{\phi}(0) \leq c_{3} \phi(0),
$$

where $c_{2}, c_{3}>0$ are constants depending on $\Omega, \rho_{1}, \rho_{2}, \theta_{1}, \theta_{2}, p, c_{v}, \mu, \nu, \varkappa$.

To extend the solution to the interval $[T, 2 T]$ we prove that

$$
\begin{aligned}
& \forall x \in \bar{\Omega}_{t}, t \in[0, T], \quad \rho_{1}<\rho(x, t)<\rho_{2}, \\
& \forall x \in \bar{\Omega}_{t}, t \in[0, T], \quad \theta_{1}<\theta(x, t)<\theta_{2} .
\end{aligned}
$$

By (2.11) and (3.7) we have

$$
\|u(t)\|_{2, \Omega}^{2}+\left\|\eta_{\sigma}(t)\right\|_{2, \Omega}^{2}+\left\|\vartheta_{\sigma}(t)\right\|_{2, \Omega}^{2} \leq \psi_{2}(A, T) \varepsilon \quad \text { for } t \leq T .
$$

Hence

$$
\sup _{\Omega^{T}}|u|^{2}+\sup _{\Omega^{T}}\left|\eta_{\sigma}\right|^{2}+\sup _{\Omega^{T}}\left|\vartheta_{\sigma}\right|^{2} \leq c(\Omega) \psi_{2}(A, T) \varepsilon,
$$

where $c(\Omega)>0$ is a constant from the imbedding theorem.

Assuming now that $\varepsilon$ is so small that

$$
\left[\frac{c_{3}}{c_{2}} c(\Omega) \psi_{2}(A, T) \varepsilon\right]^{1 / 2}<l,
$$

(where $l$ is the constant from assumption (3.8)), from estimate (3.13) we get (3.11)-(3.12). Inequalities (3.11)-(3.12) and Remark 3.2 imply estimate (3.1) with the same constants $c_{2}$ and $c_{3}$ as in (3.10). Thus the assumptions of the theorem, relations (3.11)-(3.12), Remark 3.2 and Lemmas 2.3, 3.1, 3.3 yield

$$
\bar{\phi}(t) \leq c_{3} \varepsilon \text { for } t \leq T \text {. }
$$

Hence

$$
\phi(t) \leq \frac{c_{3}}{c_{2}} \varepsilon \quad \text { for } t \leq T
$$

Therefore, in view of Theorem 2.1, Lemmas 3.5, 3.6 and estimates (2.4), (2.5), (2.8) (with the initial conditions at $T$ ), for $A$ so large that

$$
C_{1}(T)\left(\frac{c_{11} c_{3}}{c_{2}}+\alpha_{1}\right)+C_{2}(T) \alpha_{2}<A
$$

(where $c_{11}>0$ is a constant such that $\left.\left\|\left(p\left(\rho_{0}, \theta_{0}\right)-p_{0}\right) \bar{n}_{0}\right\|_{3 / 2, S}^{2} \leq c_{11} \phi(0) \leq c_{11} \varepsilon \leq c_{11}\right)$ there exists a local solution of (1.1) in the interval $[T, 2 T]$ and

$$
\begin{aligned}
& \|u\|_{\mathcal{A}_{T, \Omega_{T}}}^{2}+\left\|\vartheta_{\sigma}\right\|_{\mathcal{A}_{T, \Omega_{T}}}^{2}+\left\|\eta_{\sigma}\right\|_{\mathcal{B}_{T, \Omega_{T}}}^{2} \\
& \leq \psi_{2}(A, T)\left(\|u(T)\|_{2, \Omega_{T}}^{2}+\left\|\vartheta_{\sigma}(T)\right\|_{2, \Omega_{T}}^{2}+\left\|\eta_{\sigma}(T)\right\|_{2, \Omega_{T}}^{2}+\left\|u_{t}(T)\right\|_{1, \Omega_{T}}^{2}\right. \\
& \left.\quad+\left\|\vartheta_{\sigma t}(T)\right\|_{1, \Omega_{T}}^{2}+\left\|u_{t t}(T)\right\|_{0, \Omega_{T}}^{2}+\left\|\vartheta_{\sigma t t}(T)\right\|_{0, \Omega_{T}}^{2}\right) \leq \frac{c_{3} \psi_{2}(A, T)}{c_{2}} \varepsilon
\end{aligned}
$$

where $u, \vartheta_{\sigma}, \eta_{\sigma}$ denote $v, \theta_{\sigma}, \rho_{\sigma}$ written in the Lagrangian coordinates $\xi \in \Omega_{T}$ connected with Eulerian coordinates $x$ by the relation

$$
x=\xi+\int_{T}^{t} u\left(\xi, t^{\prime}\right) d t^{\prime}
$$

$\mathcal{A}_{T, \Omega_{T}}$ and $\mathcal{B}_{T, \Omega_{T}}$ are defined in Section 2 . 
Since by Remark 3.2 the shape of $\Omega_{t}$ does not change much for $t \leq T$, condition (3.14) is satisfied with the constant $c\left(\Omega_{T}\right)$ such that

$$
\begin{aligned}
& \sup _{\Omega_{T}}|u|^{2}+\sup _{\Omega_{T}}\left|\vartheta_{\sigma}\right|^{2}+\sup _{\Omega_{T}}\left|\eta_{\sigma}\right|^{2} \\
& \quad \leq c\left(\Omega_{T}\right)\left(\|u(t)\|_{2, \Omega_{T}}^{2}+\left\|\vartheta_{\sigma}(t)\right\|_{2, \Omega_{T}}^{2}+\left\|\eta_{\sigma}(t)\right\|_{2, \Omega_{T}}^{2}\right) \quad \text { for } T \leq t \leq 2 T .
\end{aligned}
$$

Therefore in view of (3.16) and (3.11)-(3.12) we have

$$
\forall x \in \bar{\Omega}_{t}, t \in[0,2 T], \quad \rho_{1}<\rho(x, t)<\rho_{2}
$$

and

$$
\forall x \in \bar{\Omega}_{t}, t \in[0,2 T], \quad \theta_{1}<\theta(x, t)<\theta_{2} .
$$

Now, we prove that the volume and the shape of $\Omega_{t}$ change in $[0,2 T]$ no more than they do in $[0, T]$. To do this we consider $\int_{0}^{t} v\left(x, t^{\prime}\right) d t^{\prime}$ for $0 \leq t \leq 2 T$. We estimate $\int_{0}^{T} v\left(x, t^{\prime}\right) d t^{\prime}$ by applying Lemma 3.3 and to estimate $\int_{T}^{2 T} v\left(x, t^{\prime}\right) d t^{\prime}$ we use inequality (3.16). Thus, we have

$$
\begin{aligned}
\left|\int_{0}^{t} v\left(x, t^{\prime}\right) d t^{\prime}\right| \leq \int_{0}^{T}\left|v\left(x, t^{\prime}\right)\right| d t^{\prime}+\int_{T}^{2 T} \mid u\left(\xi, t^{\prime}\right) d t^{\prime} \\
\leq T^{1 / 2}\left[c_{12}\left(\int_{0}^{T}\|v\|_{2, \Omega_{t^{\prime}}}^{2} d t^{\prime}\right)^{1 / 2}+c_{6} \frac{c_{3}}{c_{2}} \varepsilon^{1 / 2}\right] \\
\leq T^{1 / 2} \varepsilon^{1 / 2}\left(\frac{c_{12} c_{3}^{1 / 2}}{\left(c_{2} \mu_{0}\right)^{1 / 2}}+c_{6} \frac{c_{3}}{c_{2}}\right) \leq T^{1 / 2} \varepsilon^{1 / 2}\left(\frac{c_{12} c_{3}^{1 / 2}}{c_{2}^{1 / 2} \mu_{0}^{1 / 2}}+c_{6} \frac{c_{3}}{c_{2}}\right)
\end{aligned}
$$

where $c_{2}, c_{3}$ are the constants from $(3.10), \mu_{0}$ is the constant from Lemma 3.3 and $c_{6}$ is the constant from Remark 3.2.

For sufficiently small $\varepsilon$ estimates (3.17)-(3.19) imply that the differential inequality $(2.17)$ can be derived in $[T, 2 T]$ with the same constants $c_{0}, c_{1}$ and with function $\bar{\phi}(t)$ replaced by $\bar{\phi}_{T}(t)$ which has analogous form to $\phi(t)$, but the integrals over $\Omega, \tilde{\Omega}_{i}$ are replaced by integrals over $\Omega_{T}, \tilde{\Omega}_{i, T}=\left\{x: x=\xi+\int_{0}^{T} u\left(\xi, t^{\prime}\right) d t^{\prime}, \xi \in \tilde{\Omega}_{i}\right\}$, respectively.

From the forms of $\bar{\phi}(t)$ and $\bar{\phi}_{T}(t)$ it follows that

$$
\bar{\phi}_{T}(T) \leq\left[1+c_{13} T\|u\|_{\mathcal{A}_{T, \Omega}}^{2}\right] \bar{\phi}(T) .
$$

Hence by estimate (2.11), Lemma 3.3, estimate (3.10) and assumption (3.7) we get

$$
\bar{\phi}_{T}(T) \leq\left(1+c_{13} \frac{c_{3}}{c_{2}} T \psi_{2}(A, T) \varepsilon\right) c_{3} \varepsilon e^{-\mu_{0} T} .
$$

Therefore, choosing $\varepsilon$ so small that

$$
c_{14} e^{-\mu_{0} T} \equiv\left(1+c_{13} \frac{c_{3}}{c_{2}} T \psi_{2}(A, T) \varepsilon\right) e^{-\mu_{0} T}<1,
$$

we obtain

$$
\bar{\phi}_{T}(T) \leq c_{3} \varepsilon .
$$

Thus, we see that at $t=T$ functions $\bar{\phi}(t)$ and $\bar{\phi}_{T}(t)$ are estimated by the same constant $c_{3} \varepsilon$.

Next, for $\varepsilon$ sufficiently small, estimates (3.17)-(3.19) also yield inequality (3.3) for function $\bar{\phi}_{T}(t)(T \leq t \leq 2 T)$ with the same constants $c_{2}$ and $c_{3}$. Moreover, also the 
constants $c_{i}(i=4, \ldots, 12)$ and $\mu_{0}$ are the same in $[T, 2 T]$ as in $[0, T]$. Therefore, estimate (3.15) and Lemmas 2.3, 3.1 and 3.3 yield

$$
\bar{\phi}_{T}(t) \leq c_{3} \varepsilon \quad \text { for } T \leq t \leq 2 T .
$$

Hence

$$
\phi(t) \leq \frac{c_{3}}{c_{2}} \varepsilon \quad \text { for } t \leq 2 T .
$$

Estimates (3.20)-(3.21) allow to extend the solution on the interval $[2 T, 3 T]$.

Now, assume that there exists a local solution in $[0, k T]$ (where $k \geq 3$ ) satisfying

$$
\begin{gathered}
\|u\|_{\mathcal{A}_{T, \Omega_{j} T}}^{2}+\|\vartheta\|_{\mathcal{A}_{T, \Omega_{j} T}}^{2} \leq A \text { for } j=1, \ldots, k-1, \\
\|\eta\|_{\mathcal{B}_{T, \Omega_{j} T}}^{2} \leq \psi_{1}(A) \quad \text { for } j=1, \ldots, k-1, \\
\bar{\phi}_{j T}(t) \leq c_{3} \varepsilon \quad \text { for } j T \leq t \leq(j+1) T, j=0, \ldots, k-2, \\
\phi(t) \leq \frac{c_{3}}{c_{2}} \varepsilon \text { for } t \leq(k-1) T, \\
\|u\|_{\mathcal{A}_{T, \Omega_{j} T}}^{2}+\left\|\vartheta_{\sigma}\right\|_{\mathcal{A}_{T, \Omega_{j} T}}^{2}+\left\|\eta_{\sigma}\right\|_{\mathcal{B}_{T, \Omega_{j} T}}^{2} \leq \frac{c_{3}}{c_{2}} \psi_{2}(A, T) \varepsilon \text { for } j=0, \ldots, k-1,
\end{gathered}
$$

where in (3.24) by $\bar{\phi}_{j T}(T)$ we mean the function given by (2.16) with the integrals over $\Omega, \tilde{\Omega}_{i}$ replaced by integrals over $\Omega_{j T}, \tilde{\Omega}_{i, j T}$, respectively; $\bar{\phi}_{0 T}(t)=\bar{\phi}(t)$ for $t \in[0, T]$.

Moreover, assume that the volume and shape of $\Omega_{t}$ change in $[0,(k-1) T]$ no more than they do in $[0, T]$ and estimates (3.11)-(3.12) hold for $t \leq(k-1) T$ (so the constants $c_{i}, i=1, \ldots, 12$ and $\mu_{0}$ are the same in each $\left.[(i-1) T, i T], i=1, \ldots, k-1\right)$. Since the argument used to show estimates (3.11)-(3.12) for $t \leq k T$ is the same as for $t \leq T$ and for $t \leq 2 T$, to prove the existence of a local solution in $[0,(k+1) T]$ it remains to show that the volume and shape of $\Omega_{t}$ change in $[0, k T]$ no more than they do in $[0, T]$.

Applying Lemma 3.3 and estimates (3.22)-(3.26) we have, for $t \in[0, k T]$

$$
\begin{aligned}
& \left|\int_{0}^{t} v\left(x, t^{\prime}\right) d t^{\prime}\right| \leq \sum_{i=0}^{k-2} \int_{i T}^{(i+1) T}\left|v\left(x, t^{\prime}\right)\right| d t^{\prime}+\int_{(k-1) T}^{k T}\left|u\left(\xi, t^{\prime}\right)\right| d t^{\prime} \\
& \quad \leq T^{1 / 2}\left[c_{12} \sum_{i=0}^{k-2}\left(\int_{i T}^{(i+1) T} \phi\left(t^{\prime}\right) d t^{\prime}\right)^{1 / 2}+c_{6} \frac{c_{3}}{c_{2}} \varepsilon^{1 / 2}\right] \\
& \leq T^{1 / 2}\left[\frac{c_{12} c_{3}^{3 / 2}}{\left(\mu_{0} c_{2}\right)^{1 / 2}}\left(1-e^{-\mu_{0} T}\right)^{1 / 2} \sum_{i=0}^{k-2}\left(\bar{\phi}_{i T}(i T)\right)^{1 / 2}+c_{6} \frac{c_{3}}{c_{2}} \varepsilon^{1 / 2}\right] \\
& \quad \leq T^{1 / 2}\left\{\frac{c_{12} c_{3}^{3 / 2}}{\left(\mu_{0} c_{2}\right)^{1 / 2}}\left(1-e^{-\mu_{0} T}\right)^{1 / 2}\left[\bar{\phi}(0)\left(1+c_{14} e^{-\mu_{0} T}+c_{14}^{2} e^{-2 \mu_{0} T}+\ldots\right)\right]^{1 / 2}\right. \\
& \left.\quad+c_{6} \frac{c_{3}}{c_{2}} \varepsilon^{1 / 2}\right\} \leq T^{1 / 2} \varepsilon^{1 / 2}\left(\frac{c_{12} c_{3}^{3 / 2}}{\mu_{0}^{1 / 2} c_{2}^{1 / 2}}\left(\frac{1-e^{-\mu_{0} T}}{1-c_{14} e^{-\mu_{0} T}}\right)^{1 / 2}+c_{6} \frac{c_{3}}{c_{2}}\right),
\end{aligned}
$$

where $c_{2}$ and $c_{3}$ are the constants from (3.10), $\mu_{0}$ is the constant from Lemma 3.3 and $c_{6}$ is the constant from Remark 3.2.

Thus, the right-hand side of (3.27) does not depend on $k$. Therefore, for $\varepsilon$ sufficiently small the shape of $\Omega_{t}$ change in $[0, k T]$ no more than it does in $[0, T]$ and the constants $c_{i}(i=1, \ldots, 13)$ and $\mu_{0}$ are the same in each $[i T,(i+1) T]$ for $i=0, \ldots, k-1$. 
Estimates (3.24)-(3.25), (3.27) and Lemmas 2.3, 3.1 and 3.3 give $\bar{\phi}_{(k-1) T}(t) \leq c_{3} \varepsilon$ for $(k-1) T \leq t \leq k T$ and $\phi(t) \leq \frac{c_{3}}{c_{2}} \varepsilon$ for $t \leq k T$.

This completes the proof of the theorem.

\section{References}

[1] L. Landau, E. Lifschitz, Hydrodynamics, Nauka, Moscow 1986 (in Russian).

[2] J. Serrin, Mathematical principles of classical fluid mechanics, in: Handbuch der Physik, Bd. VIII/1, Springer, Berlin, Göttingen, Heidelberg, 1959, 125-263.

[3] V. A. Solonnikov, Solvability of the evolution problem for an isolated amount of a viscous incompressible capillary fluid, Zap. Nauchn. Sem. Leningrad. Otdel. Mat. Inst. Steklov. (LOMI) 140 (1984), 179-186 (in Russian); English transl.: J. Soviet Math. 32 (1986), 223-238.

[4] V. A. Solonnikov, On an unsteady flow of a finite mass of a liquid bounded by a free surface, Zap. Nauchn. Sem. Leningrad. Otdel. Mat. Inst. Steklov. (LOMI) 152 (1986), 137-157 (in Russian); English transl: J. Soviet Math. 40 (1988), 672-688.

[5] V. A. Solonnikov, On an unsteady motion of an isolated volume of a viscous incompressible fluid, Izv. Akad. Nauk SSSR Ser. Mat. 51 (1987), 1065-1087 (in Russian); English transl.: Math. USSR-Izv. 31 (1988), 381-405.

[6] V. A. Solonnikov, A. TANi, Evolution free boundary problem for equations of motion of viscous compressible barotropic liquid, in: The Navier-Stokes Equations II-Theory and Numerical Methods (Oberwolfach, 1991), Lecture Notes in Math. 1530, Springer, Berlin, 1992, 30-55.

[7] E. ZADRZYŃSKA, On nonstationary motion of a fixed mass of a general viscous compressible heat conducting capillary fluid bounded by a free boundary, Appl. Math. (Warsaw) 25 (1999), 489-511.

[8] E. ZADRZYŃSKA, Evolution free boundary problem for equations of viscous compressible heat-conducting capillary fluids, Math. Methods Appl. Sci. 24 (2001), 713-743.

[9] E. ZADRZYŃSKA, W. M. ZAJĄCZKOWSKI, On global motion of a compressible viscous heat-conducting fluid bounded by a free surface, Acta Appl. Math. 37 (1994), 221-231.

[10] E. ZADRZYŃSKA, W. M. ZAJĄCZKOWSKI, On the global existence theorem for a free boundary problem for equations of a viscous compressible heat conducting fluid, Ann. Polon. Math. 63 (1996), 199-221.

[11] E. ZADRZYŃSKA, W. M. ZAJĄCZKOWSKI, Local existence of solutions of a free boundary problem for equations of compressible viscous heat-conducting fluids, Appl. Math. (Warsaw) 25 (1998), 179-220.

[12] E. ZAdRZyŃSKA, W. M. ZAJĄCZKOWski, On nonstationary motion of a fixed mass of a viscous compressible barotropic fluid bounded by a free boundary, Colloq. Math. 79 (1999), 283-310.

[13] E. Zadrzyńska, W. M. ZAJĄCZKOWski, Differential inequalities for general fluid motions bounded by a free surface, to be published.

[14] W. M. ZAJACCZKOWSKI, On nonstationary motion of a compressible barotropic viscous fluid bounded by a free surface, Dissertationes Math. (Rozprawy Mat.) 324 (1993), 101 pp.

[15] W. M. ZAJĄCZKOWSKI, On nonstationary motion of a compressible barotropic viscous capillary fluid bounded by a free surface, SIAM J. Math. Anal. 25 (1994), 1-84. 